\title{
SPRACHE ODER SPRACHFORM ZU EINER THEORIE DER SCHRIFTLICHKEIT
}

0 . Vorbemerkung

1. Die strukturelle Konzeption der Glossematiker

1.1. Zur Konzeption

1.2. Die Grenzen der Anwendbarkeit

1.3. Zwei Voraussetzungen für die Anwendung

2. Die funktionalistische Konzeption

2.1. Zur Bestimmung der Funktionen

2.2. Zur Bestimmung des Verhältnisses der Sprachfunktionen untereinander

2.3. Die Grundlage der Unterscheidung

3. Eine pragmatische Konzeption

3.1. Die Grundlage

3.2. Die Unterscheidung

3.3. Der Geltungsbereich der Unterscheidung

3.4. Einige Konsequenzen

\section{Vorbemerkung}

Schriftlichkeit kann unter verschiedenen Gesichtspunkten untersucht werden: historischen, kulturwissenschaftlichen, soziologischen, psychologischen, pädagogischen und selbstverständlich auch linguistischen. Im folgenden soll es ausschließlich um linguistisch relevante Aspekte von Schriftlichkeit gehen.

Eine Theorie der linguistisch relevanten Aspekte von Schriftlichkeit steht uns ebensowenig zur Verfügung wie ein Theorie von Schriftlichkeit überhaupt. Wie weit wir von einem solchen Ziel entfernt sind, zeigt allein schon die Tatsache, daß wir noch nicht einmal wissen, wie wir die linguistisch relevanten Aspekte von Schriftlichkeit bezeichnen sollen. Meistens spricht man von ,geschriebener Sprache". Gegen eine solche Bezeichnung wäre aber einzuwenden, daß sie einem Mißverständnis Vorschub leisten könnte. Denn der Ausdruck „geschrieben“ legt es nahe, an den Prozeß des Schreibens und also an den Vorgang der Produktion schriftlicher Äußerungen (Texte) zu denken. Geschriebene Sprache wäre demnach die sprachliche Gestalt des aus diesem Prozeß hervorgegangenen Produktes: die sprachliche Gestalt einer schriftlichen Äußerung, und zwar einer jeden schriftlichen Äußerung, sei es ein genuin schriftlicher Text, sei es die Transkription einer mündlichen Äußerung, was gewiß nicht gemeint sein kann.

Ich habe an anderer Stelle (O. Ludwig 1983) vorgeschlagen, statt von „geschriebener“ von „schriftlicher Sprache“ zu sprechen. Eine solche Bezeichnung macht deutlicher, daß es sich bei den linguistisch relevanten Aspekten von Schriftlichkeit in erster Linie um Eigenschaften von Sprache (und nicht 
von schriftlichen Äußerungen) handelt. Sie hat zudem den Vorteil, daß sie sich gut als Oberbegriff für die Sprache verschiedener Modi der schriftlichen Produktion eignet: die handgeschriebene, die maschinengeschriebene, die gedruckte, die durch den Computer hergestellte - lauter Varianten schriftlicher Sprache, die bisher so gut wie überhaupt nicht untersucht worden sind.

Jede Theorie der linguistisch relevanten Aspekte von Schriftlichkeit oder - wie es im folgenden der Kürze wegen heißen soll - jede Theorie der schriftlichen Sprache hat es vorrangig mit deren Differenzierung von der mündlichen Sprache zu tun. Dabei geht es um ein ganzes Bündel von Fragen, von denen die wichtigsten kurz angeführt seien.

1. Auf welcher Dimension von Sprache soll die Differenzierung vorgenommen werden? In der sprachwissenschaftlichen Diskussion haben drei Dimensionen eine Rolle gespielt: die Substanzen, die Funktionen und die sprachlichen Formen. Die Substanzen an sich sind aber linguistisch nicht relevant, relevant sind dagegen allein ihre sprachlichen Auswirkungen. An „Funktionen“ der schriftlichen Sprache werden immer wieder ihre Visualität, ihre Bewahrbarkeit und ihre Transportabilität genannt. Nun sind das aber eher durch die graphische Substanz bedingte Eigenschaften oder Fähigkeiten schriftlicher Äußerungen (Texte) und nicht solche der schriftlichen Sprache. Bleibt noch die Dimension der sprachlichen Formen. Für sie müssen aber, wie ich noch ausführen werde, besondere Bedingungen vorliegen, damit auf dieser Dimension eine Differenzierung der schriftlichen von der mündlichen Sprache durchgeführt werden kann.

2. Wenn eine Differenzierung auf der Dimension der sprachlichen Formen vorgenommen wird, dann stellt sich die Frage nach dem Geltungsbereich. Die Kardinalfrage lautet in diesem Zusammenhang: Gibt es oberhalb der phonographischen Ebene, deren Differenzen offensichtlich sind, noch weitere sprachliche Unterschiede und kann es sie überhaupt geben? Eine nicht unerhebliche Zahl von Sprachwissenschaftlern hält das schlicht für ausgeschlossen. Andere sind vorsichtiger, schließen Besonderheiten der schriftlichen Sprache im Morphologischen, Syntaktischen oder Lexikalischen nicht von vornherein aus, möchten diese aber als eine „quantité négligable“ betrachtet wissen. Ein Beispiel:

eine Syntax einer Sprache ist grundsätzlich für beide Ausdrucksebenen dieselbe Besonderheiten einer „Syntax der gesprochenen (resp. geschriebenen) Sprache“ sind (heterogene) Akzidentien, die im übrigen nur in bezug auf eine „Normalform“ der Syntax als Besonderheiten charakterisierbar sind. Dasselbe gilt für andere Ebenen der Grammatik oberhalb der Phonologie (H. Glück 1987: 37).

Nur wenige sehen hier ein Problem. Auch dafür ein Beispiel:

Es ist weitgehend ungeklärt, inwieweit schriftliche Sprache gegenüber mündlicher Sprache spezifische (...) Eigenheiten in einem anderen Teilsystem aufweist, z. B. in der Syntax oder im Wortschatz. Ganz offensichtlich ist z. B. die Interpunktion im Deutschen nach rein syntaktischen Kriterien geregelt - aber die syntaktischen Regeln sind nicht am Schriftsystem orientiert. Ein anderer Fall sind Phänomene 
wie der Gebrauch des Passé Simple im Französischen, das nur in der schriftlichen Sprache verwendet wird. Dennoch ist es wohl falsch zu sagen, das Passé Simple zeige, daß die schriftliche Sprache im Französischen eine eigene Syntax und Morphologie aufweist. Derlei Diskrepanzen zwischen gesprochener und geschriebener Sprache sind noch erheblich größer im Arabischen, wo man sagen muß, daß die schriftliche Form einen Dialekt betrifft, der nicht (mehr) gesprochen wird. Es scheint mir klar, daß die Problematik noch nicht ausdiskutiert ist. (H. Günther 1988: 41).

3. In welchem Sinne können schriftliche und mündliche Sprache als zusammengehörig betrachtet werden? Es ist dies die Frage nach der Voraussetzung der Differenzierung oder nach deren Grundlage. Oft wird ein Zusammenhang hergestellt, dadurch daß man annimmt, daß beiden eine einheitliches Sprachsystem zugrunde läge, deren Realisierung, Aktualisierung oder Manifestierung sie darstellten. Ein Zusammenhang kann aber auch dadurch hergestellt werden, daß man schriftliche und mündliche Sprache für zwei Teile eines Ganzen hält, deren Koordination erst die Totalität einer Sprache ergäbe. In beiden Fällen wäre die Zusammengehörigkeit durch die sprachliche Struktur bedingt. Es ist aber auch der Fall nicht auszuschließen, daß in einer Sprache gesprochen und in einer anderen Sprache geschrieben wird. In einem solchen Fall würde der Zusammenhang durch die Menschen hergestellt, die sich der Sprachen bedienen. Eine formale Zusammengehörigkeit wäre also nicht in jedem Falle erforderlich (dazu ausführlicher unten).

Nach den drei angeführten Gesichtspunkten sollen im folgenden drei Konzeptionen von schriftlicher Sprache erörtert werden:

- eine strukturalistische: die der Glossematiker

- eine funktionalistische: die von Josef Vachek, der sich mit den angeschnittenen Fragen bisher am ausführlichsten beschäftigt hat, und

- eine pragmatische, die hier zur Diskussion gestellt wird.

\section{Die strukturelle Konzeption der Glossematiker}

\subsection{Zur Konzeption}

Die glossematische Konzeption zeichnet sich dadurch aus, daß die Unterscheidung zwischen schriftlicher und mündlicher Sprache strikt auf die phonographische Ebene beschränkt bleibt.

Die Begründung erfolgt in zwei Schritten (zum folgenden vgl. L. Hjelmslev 1943/1961: 47-60; H. J. Uldall 1949; H. Glück 1987: 22 f., 36 ff., 41 ff.):

(1) Dadurch daß nicht nur auf der Inhaltsebene, sondern auch auf der Ausdrucksebene zwischen Form und Substanz unterschieden wird, ist es möglich, die Ausdrucksebene doppelt zu fassen und auf ihr sowohl zwei Substanzen (die phonische und die graphische Substanz) als auch zwei Formen anzusetzen:

ebenso wie die phonologische Komponente die eine Form des Ausdrucks aller höheren Hierarchisierungsebenen darstellt und von den Regularitäten jeder der anderen Komponenten beeinflußt ist, stellt die graphematische Komponente die 
ande re Form des Ausdrucks aller höheren Hierarchisierungsebenen dar und ist entsprechenden Einwirkungen ausgesetzt (H. Glück 1987: 41).

(2) Dadurch daß strikt zwischen Inhalts- und Ausdrucksebene unterschieden wird, können die Unterschiede auf der Ausdrucksebene nicht auf die Inhaltsebene durchschlagen. Schon 1943 hatte L. Hjelmslev ausdrücklich die Auffassung zurückgewiesen:

that a different "substance" is accompanied (...) by a changed linguistic form (L. Hjelmslev 1943/1961: 104).

Der Geltungsbereich der Unterscheidung wird damit auf die Form der bedeutungsdifferenzierenden sprachlichen Einheiten beschränkt. Auf die Form der bedeutungstragenden Einheiten hat sie keinen Einfluß.

Im Rahmen der glossematischen Sprachtheorie ist es dann auch nicht mehr möglich, von einer schriftlichen Sprache zu sprechen. Es gibt hier nur ein und dieselbe Sprache, und diese ist sowohl für das Schriftliche wie auch für das Mündliche gleich. Rede und Schrift, so H. J. Uldall, sind

expressions of one and the same language (1939/1949: 11).

Unterschiede im Sprachgebrauch, die durchaus zugestanden werden, berühren nicht, so Hjelmslev, das Sprachsystem:

Thus, various phonetic usages and various written usages can be ordered to the expression system of one and the same linguistic schema (1943/1961: 105).

Und so gibt es auch keinen Anlaß, in der grammatischen Beschreibung auf unterschiedliche Realisierungsweisen Rücksicht zu nehmen:

eine Syntax einer Sprache ist grundsätzlich für beide Ausdrucksebenen dieselbe (...). Dasselbe gilt für andere Ebenen der Crammatik oberhalb der Phonologie (H. Glück 1987: 37).

Damit ist auch klar, auf welcher Grundlage die Differenzierung vorgenommen wird. Sinnvoll ist eine solche nur im Rahmen und im Zusammenhang ein und dersclben Sprache. Es gibt nach der glossematischen Theorie zwar zwei Ausdrucksformen, eine geschriebene und eine gesprochene, aber nur eine Sprache. Es ist darum nicht mehr als konsequent, wenn Helmut Glück nicht von „schriftlicher (oder geschriebener)“ und „mündlicher (oder gesprochener) Sprache" spricht, sondern den für die glossematische Konzeption treffenden Ausdruck ,geschriebene bzw. gesprochene Sprachform“ benutzt.

Ich möchte im folgenden nicht auf die internen Probleme der glossematischen Konzeption eingehen, stattdessen die Frage nach den Realitäten stellen, auf die eine solche Theorie der schriftlichen Sprachform, wenn sie denn in sich schlüssig sein sollte, überhaupt anwendbar wäre und welche Bedingungen gegeben sein müssen, damit eine solche Anwendung durchgeführt werden kann.

\subsection{Die Grenzen der Anwendbarkeit}

Da die Differenzierung von schriftlicher und mündlicher Form nur innerhalb ein und derselben Sprache möglich ist, läßt sich die glossematische Konzeption grundsätzlich nicht auf die Fälle anwenden, in denen Menschen 
in verschiedenen Sprachen sprechen und schreiben (vgl. Hinweise bei N. Catach 1987: $170 \mathrm{f}$. und W. Klein 1985: 28). Damit ist eine große Zahl sprachlicher Situationen ausgeschlossen. Es handelt sich um ausgesprochen diglottische Situationen (zur Diglossie vgl. G. Lüdi im Druck). Diglossie kann unterschiedlich bedingt sein und sich infolgedessen auch unterschiedlich darstellen.

Für das Verhältnis von schriftlicher und mündlicher Sprache scheinen drei Situationen typisch zu sein.

(1) Schriftliche und mündliche Sprache unterscheiden sich grundsätzlich

$\mathrm{Da} ß$ es Völker gibt, die in einer Sprache sprechen, aber in einer ganz anderen schreiben, mag uns Westeuropäern erstaunlich vorkommen, ungewöhnlich ist es indessen nicht.

Als die Japaner im 6. oder 7. Jahrhundert n. Chr. zu schreiben anfingen, übernahmen sie aus China, dem damals in Ostasien kulturell führenden Land, mit der Schrift zugleich auch die zu dieser Schrift gehörige Sprache. Sie sprachen also das Japanische, schrieben aber Chinesisch. So blieb das viele Jahrhunderte (vgl. F. Coulmas 1989: 122-133). Durchaus kein Einzelfall. Auch die Koreaner und die Vietnamesen haben ihre Schrift aus China entlehnt und, wenn sie schrieben, die chinesische Sprache verwendet (ebd.: 113 und 115). Die Verhältnisse, die wir in Ostasien finden, lassen sich ohne weiteres verallgemeinern:

Whenever illiterate peoples wanted to write, they usually borrowed the written language of an neighboring people (ebd.: 43)

So haben die Assyrer und Babylonier zuerst einmal in sumerischer Sprache geschrieben (ebd.: 80). Dasselbe gilt auch für die Hettiter (ebd.: 43). In Europa war es nicht anders. Die Mönche, die als erste im 9. Jahrhundert die Schrft nach Westeuropa brachten, haben nicht nur das lateinische Alphabet verwendet, sondern auch in lateinischer Sprache geschrieben, und so ist das Lateinische das ganze Mittelalter hindurch die Sprache fürs Schreiben geblieben (vgl. M. T. Clanchy 1979; P. Kopsch 1982; P. Burke 1987/1989). Heute können wir ähnliche Verhältnisse in allen Teilen Afrikas beobachten. Man spricht in einer der vielen Stammessprachen, schreibt aber in der Sprache der ehemaligen Kolonialherren:

not only as a relict of colonial rule but because the adoption of a metropolian language for written and much oral communication avoids to choose one local language among many, which would create overwhelming political and cultural problems (J. Goody 1987: 281).

$\mathrm{Zu}$ allen Zeiten, seit Menschen gelernt haben zu schreiben, und überall, wo geschrieben worden ist, hat es also den Fall gegeben, daß für das Schreiben eine Sprache Verwendung fand, die grundsätzlich nichts mit der Sprache zu tun hatte, die man Tag für Tag beim Sprechen gebrauchte. Hier handelt es sich wirklich um zwei grundverschiedene Sprachen. Wie aber, wenn die schriftliche und die mündliche Sprache doch etwas miteinander zu tun haben? Auch 
dann kann man unter bestimmten Umständen durchaus von zwei Sprachen sprechen.

(2) Man spricht und schreibt zwar in derselben Sprache, doch die schriftliche Sprache kann nicht als schriftliche Form der mündlichen Sprache angesehen werden.

Der Fall ist nicht ungewöhnlich, daß schriftliche und mündliche Sprache Varietäten ein und derselben Sprache sind, die schriftliche Sprache aber als schriftliche Sprachform nicht der mündlich gebrauchten Varietät zugeordnet werden kann. Einen solchen Fall finden wir z. B. in der deutschsprechenden Schweiz (vgl. P. Kühn 1980; R. E. Keller 1982; P. Sieber und H. Sitta 1986; G. Lüdi im Druck). Man spricht „Schwyzertütsch“. (,Schwyzertütsch“ ist ein Sammelbegriff für "ein Konglomerat von regionalen und lokalen Dialekten“ (G. Lüdi).) Eine schriftliche Form des Schwyzertütschen hat sich aber nicht durchsetzen können. Stattdessen benutzen die deutschsprechenden Schweizer, wenn sie schreiben, die deutsche Standardsprache, genauer die schriftliche Form der deutschen Standardsprache. Das „Schriftdeutsch“ der Schweizer ist also nicht die schriftliche Form des „Schwyzertütschen“. „Zweisprachigkeit in der einen - deutschen - Sprache", auf diese Formel haben Peter Sieber und Horst Sitta die Verhältnisse in der deutschsprachigen Schweiz gebracht (1986: 17).

(3) Schriftliche und mündliche Sprache repräsentieren unterschiedliche Entwicklungsstufen ein und derselben Sprache

Die Tendenz, sprachliche Ausdrücke auch dann noch zu verwenden, wenn sie in der mündlichen Sprache längst aus der Mode gekommen sind, kann dazu führen, daß die schriftliche Sprache mit der Entwicklung der mündlichen nicht Schritt hält und so schließlich eine Entwicklungsstufe repräsentiert, die die mündliche Sprache längst verlassen hat. In einem solchen Fall kann die schriftliche Sprache nicht als schriftliche Form der gleichzeitig mündlich verwendeten Sprachform angesehen werden. Zwei Sprachen, die zu den verbreitetsten auf der Welt überhaupt zählen, weisen solche Verhältnisse auf: das Chinesische und das Arabische (vgl. F. Coulmas 1981: 99 ff.; Ch. N. Li und S. A. Thompson 1982: 84; T. F. Mitchell 1982).

In den angeführten Fällen von Diglossie dürfte sich die Anwendung der glossematischen Konzeption der schriftlichen Sprachform grundsätzlich verbieten. Wie aber steht es mit den nicht-diglottischen Verhältnissen, für die allein diese Konzeption entwickelt worden ist?

\subsection{Zwei Voraussetzungen für die Anwendung}

Auch in nicht-diglottischen Situationen ist die glossematische Konzeption nicht ohne weiteres anwendbar. Es müssen mindestens zwei Voraussetzungen erfüllt sein: 
- Aus dem Bereich der schriftlichen Sprache müssen die Zahlen ausgeschlossen werden. (1)

- Andere Abweichungen der schriftlichen Sprache müssen als akzidentiell betrachtet werden können. (2)

\section{(1) Der Bereich der Zahlen}

Für die mündliche Sprache werden gewöhnlich keine Unterschiede zwischen den Zahlen und anderen lexikalischen Einheiten gemacht. Die Zahlen gehören wie selbstverständlich zu diesen. Für die schriftliche Sprache ist dies keineswegs so selbstverständlich.

Eine solche Einschätzung der Zahlen geschieht nicht ohne Grund. Denn in der Tat werden die Zahlen in der schriftlichen Sprache anders als in der mündlichen behandelt. Der Unterschied betrifft nicht nur die Schreibung, d. h. die Tatsache, daß Zahlen sowohl analog zur mündlichen Sprache, alphabetisch (,eins“, „Zwei“, „drei“, „zehn“, „zwanzig“, „dreißig“ usw.) als auch logographisch (,1“, „,"“, „3“, „10“, ,20“, ,30“ usw.) ausgedrückt werden können, sondern vor allem die Bildung der mehrstelligen Zahlen (zum folgenden vgl. G. Ifrah 1981/1989: 411 ff.; W. Haas 1968):

1. In der mündlichen Sprache werden die mehrstelligen Zahlen morphologisch gebildet („fünf“, „,ünf-zig“, „fünf-hundert“ usw.), in der schriftlichen Sprache dagegen durch die Position der Ziffern (, ,“", „50“, „500“ usw.). Man spricht darum vom „Positionsprinzip“ oder auch von einer "Stellenwertschrift" (G. Ifrah).

2. In der mündlichen Sprache des Deutschen werden die Zehner von rechts nach links (,zweiundfünfzig“), alle Zahlen über hundert aber von links nach rechts gebildet (,zweitausenddreihundertfünfzig"). Aufgeschrieben werden die mehrstelligen Zahlen von links nach rechts, und zwar alle, doch gebildet, d. h. gezählt, werden sie in der umgekehrten Richtung:

The Arabic notation (...) constructs its complex names in the opposite direction, i.e. in ascending order of magnitude; and the order is, itself, distinctive. This difference is generally hidden from us by the facility we acquire in translating from, or into spoken numerals. Only when this facility fails us, as happens when we are faced with a very long Arabic numeral - say, 2804643597 -, then the written numeral's own syntactic construction is acknowledged. (...) This has to be done by beginning with the units and procedding, from right to left, in ascending order of magnitude; we place the "thousand"-marker after three digits, „million“ after six, until finally, after nine, we are ready to reverse the order and to begin our translation with "two billion“. (W. Haas 1988: $208 \mathrm{f}$.)

3. Für die bedeutsame Rolle, die die Ziffer „, “ in der schriftlichen Sprache zur Kennzeichnung einer nicht besetzten Position spielt, gibt es in der mündlichen überhaupt keine Entsprechung (,10“, „100“, „1000“, aber „zehn“, „hundert“, „tausend“ usw.). Das gilt allerdings nur für die Bildung arabischer Zahlen, nicht für die römischen Zahlen (X, C, M etc.).

Wenn auch die Schreibung der Zahlen teilweise und die Bildung mehrstelliger Zahlen in der schriftlichen Sprache erheblich von denen in der mündlichen 
Sprache abweicht, so gibt es doch nicht den geringsten Grund, die Zahlen anders als in der mündlichen Sprache - aus dem Geltungsbereich der schriftlichen Sprache herauszunehmen. Aber genau dies wäre erforderlich, wenn man die glossematische Konzeption anwenden wollte.

\section{(2) Die sogenannten Akzidentien}

In allen mir bekannten Sprachen gibt es über die Zahlen hinaus Besonderheiten der schriftlichen Sprache:

- im Deutschen: etwa die Verwendung einiger Deiktika („oben“ und „unten“, „hier“ und ,,jetzt“ in Büchern), die Zulässigkeit einiger Ellipsen (,im Rahmen der Literaturwissenschaft und -didaktik"), neuerdings die Verwendung des großen „I“ in Personenbezeichnungen u. a.m.

- im Französischen: die Verwendung des passé simple.

Nur unter der Voraussetzung, daß solche Besonderheiten der schriftlichen Sprache als „Akzidentien“ (H. Glück) betrachtet und also vernachlässigt werden können, wäre eine glossematische Konzeption auf die angeführten Sprachen anwendbar. Dagegen aber lassen sich zwei Einwände vorbringen. Erstens sind diese Sprachen nie systematisch auf solche Besonderheiten hin untersucht worden, so daß wir nicht einmal abschätzen können, in welchem Ausmaße sie anzutreffen sind. Zweitens ist der theoretische Status solcher Besonderheiten nicht geklärt. Daß man sie ohne weiteres in der grammatischen Beschreibung berücksichtigen kann (H. Glück 1987: 37), ändert nichts an diesem Mangel.

Nimmt man nun alle besprochenen Fälle zusammen, die mit und die ohne Diglossie, dann wird deutlich, daß die Anwendungsmöglichkeiten der glossematischen Konzeption recht beschränkt und daß die Fälle, auf die sie unter Umständen angewendet werden könnte, nicht unproblematisch sind.

\section{Die funktionalistische Konzeption}

Für die funktionalistische Konzeption ist kennzeichnend, daß die Unterscheidung zwischen schriftlicher und mündlicher Sprache nicht, wie bei den Glossematikern, auf die Dimension der Substanzen, sondern auf die der Funktionen gegründet wird. Das heißt nicht, daß die Unterschiede in den Substanzen ignoriert würden. Ihre Bedeutung für die Ausbildung etwa der schriftlichen Sprache wird durchaus anerkannt (vgl. J. Vachek 1939/1976: 236; 1949/1967: 153; 1973/1976: 245 u. ö.). Priorität aber haben die funktionalen Unterschiede:

To the difference of material existing between the two is added another difference, more profound and more essential, that is to say, a difference of functions (1949/ 1976: 153)

In diesem Punkt ist die funktionalistische Konzeption klar und eindeutig, in anderen gibt es einige Ungereimtheiten. Unklar ist schon die Beantwortung der Frage, um welche Funktionen es sich eigentlich handele. 


\subsection{Zur Bestimmung der Funktionen}

$\mathrm{Zu}$ den Funktionen von schriftlicher und mündlicher Sprache hatte sich Josef Vachek 1939 so geäußert:

Die Aufgabe einer Sprechäußerung besteht darin, auf eine Tatsache möglichst unmittelbar zu reagieren; eine Schriftäußerung dagegen soll die Stellungnahme zu einem Sachverhalt in möglichst dauerhafter Weise festlegen (1939/1976: 232f.)

Diese Bestimmung ist im Verlauf der Zeit differenziert worden und hat schließlich die folgende kanonische Form erhalten:

The spoken norm of language may be defined as a system of phonologically implementable language elements whose distinctive function it is to react to a given stimulus (which, as a rule, is an urgent one) in a dynamic way, i.e. promptly and immediately, and so as to express not only the purely congnitive but also the emotional reaction of the language user.

The written norm, on the other hand, is a system of graphically implementable language elements whose distinctive function it is to react to a given stimulus (which, as a rule, is not an urgent one) in a static way, i. e. in a preservable and easily surveyable manner, concentrating particularly on the purely cognitive reaction of the language user (1982: $44 \mathrm{f}$.)

Diese Bestimmungen sind in der wissenschaftlichen Literatur oft wiederholt, zuweilen auch modifiziert, aber grundsätzlich nicht in Frage gestellt worden (vgl. jedoch M. Kohrt 1987: 206-269). Es stellen sich aber Fragen.

Von Anfang an hat Josef Vachek klar und deutlich zwischen schriftlicher Sprache und schriftlichen Äußerungen unterschieden:

Unter geschriebener Sprache verstehen wir eine Norm, oder besser ein System von graphischen (bzw. typographischen) Mitteln, die innerhalb einer Gemeinschaft als Norm anerkannt werden. Die Schriftäußerungen dagegen sind einzelne konkrete Realisierungen der besagten Norm (1939/1976: 230; vgl. auch 1949/1976: 153; 1965 : 960 u. ö.).

Die Unterscheidung entspricht in etwa der von "langue“ und „parole“ (vgl. 1939/1976: 230 f.). Es ist dies die Unterscheidung zwischen dem Produkt (den Schriftäußerungen) und den Mitteln, durch die das Produkt (neben anderem) zustandekommt: dem System der schriftlichen Sprache. Nun sollte man annehmen, daß, wenn schon zwischen schriftlicher Sprache und Schriftäußerungen unterschieden wird, dann konsequenterweise auch zwischen den Funktionen von Schriftäußerungen und schriftlicher Sprache. Das ist aber nicht geschehen (vgl. außer den angeführten Stelle auch 1973/1976: 245 f., 1981: $180 \mathrm{f}$. u. ö.).

Problematisch ist eine solche Bestimmung der Funktion der schriftlichen Sprache deshalb, weil die Definition der Funktion von schriftlichen Äußerungen auf die Definition der Funktion von schriftlicher Sprache übertragen wird, so da $\beta$ beide nun identisch sind, ohne da $\beta$ auch nur ein einziges Wort darüber verloren wird, warum das so sein muß.

Es kommt aber noch eine weitere Schwierigkeit hinzu: die Bestimmung der Funktion schriftlicher Äußerungen. Was könnte das für eine Funktion 
sein? Kein Zweifel, daß einzelne, konkrete Schriftstücke eine Funktion haben: der Brief an einen Freund; der Artikel, an dem ich gerade arbeite; die Steuererklärung usw. Auch ganze Sorten von Schriftstücken, wie etwa die wissenschaftliche Abhandlung, das Telegramm oder der Personalausweis, werden nicht nur durch ihre Form, sondern auch durch ihre Funktion definiert. Also kann man auch in diesem Fall noch von einer Funktion sprechen. Wie aber steht es, wenn alle möglichen Schriftstücke zusammengenommen werden, wie Josef Vachek das tut? Kann man auch dann noch von einer Funktion sprechen? Lassen sich überhaupt so verschiedenartige schriftliche Äußerungen auf einen Nenner bringen, wie die flüchtige Notiz auf einem Zettel, das mehrbändige Lexikon im Bücherschrank, ein Beleidsschreiben, die Rechnung des Elektrizitätswerkes, der Klappentext auf einem Buchumschlag, die Liste aller Monatsnamen? Ich denke, daß das kaum möglich sein wird, auch dann nicht, wenn die Bestimmung, die man findet, so abstrakt wie nur irgendmöglich gehalten wird. So gibt es vermutlich keinen Sinn, von der einen Funktion der Schriftsprache oder der schriftlichen Äußerungen zu reden.

$\mathrm{Zu}$ den beiden angeführten Schwierigkeiten kommt noch eine weitere hinzu: die Bestimmung des Verhältnisses der Funktionen von schriftlicher und mündlicher Sprache untereinander.

\subsection{Zur Bestimmung des Verhältnisses der Sprachfunktionen untereinander}

Es gibt in den Schriften von Josef Vachek zwei Arten der Bestimmungen des Verhältnisses: umgangssprachliche Beschreibungen auf der einen Seite und Versuche, das Verhältnis auf wissenschaftliche Begriffe zu bringen, auf der anderen.

In den umgangssprachlichen Beschreibungen werden zwei Tatsachen hervorgehoben:

1. Es gibt unterschiedliche Bedürfnisse der Menschen hinsichtlich ihrer Kommunikation. Einige können besser durch schriftliche, andere besser durch mündliche Äußerungen befriedigt werden:

as a rule, in some situations it is the spoken utterances that can serve the communicational intentions of the language user more efficiently than their written counterparts, in other situations, again, the written utterances will prove to be much more efficient (1973/1976: 48 u. ö.)

2. Die Situationen, in denen vorzugsweise schriftliche Äußerungen Verwendung finden, zeichnen sich dadurch aus, daß sie oft „höheren kulturellen und/oder zivilisatorischen Zwecken und Funktionen“" (1973/1976: 246) dienen:

that the written norm obviously caters for higher, that is more specialized, cultural and civilizational needs of the language community than its spoken counterpart (1973: 49).

In den Versuchen, das Verhältnis auf einen Begriff zu bringen, geht es um „logische Schlußfolgerungen“, wie Josef Vachek meint (1973/1976: 246). Aus der Tatsache, daß es Situationen gibt, in denen die Verwendung schriftlicher 
Äußerungen angemessener ist, wird geschlossen, daß sich die Funktionen von schriftlichen und mündlichen Äußerungen (und also auch von schriftlicher und mündlicher Norm) komplementär verteilen:

Jedes Glied der Sprachgemeinschaft ist sich dessen bewußt, daß die beiden Normen komplementär sind, da jede von beiden eine spezifische Funktion hat, in welcher sie von der anderen nicht gut vertreten werden kann (1939/1976: 233, vgl. auch 1973: 48; 1973/1976: 246; 1982: 45).

Nun ist aber eine komplementäre Verteilung etwas anderes als ein höherer Grad an Effizienz. Im einen Fall handelt es sich um ein graduelles Verhältnis, im anderen Fall um ein Verhältnis zweier sich gegenseitig ausschließender Teile.

Aus der Tatsache, daß die schriftliche Sprache in Situationen verwendet wird, die „höheren kulturellen und zivilisatorischen Zwecken“ dienen, wird gefolgert, daß sie Glied in einer Opposition ist, und zwar das merkmalhaltige Glied:

All das läuft (...) darauf hinaus, daß die geschriebene Norm als das merkmalhaltige Glied einer Opposition zu charakterisieren ist, deren merkmalloses Glied die entsprechende gesprochene Norm darstellt (1973/1976: 246; vgl. auch 1973: 49).

Hier wird aus einem hierarchischen Verhältnis eine logische Opposition gemacht.

In den „logischen Schlußfolgerungen“ kommen zwei scheinbar widersprechende, in Wirklichkeit aber einander ergänzende Tendenzen zum Ausdruck. Auf der einen Seite die Tendenz, die Funktionen von schriftlicher und mündlicher Sprache zu polarisieren und damit einen Gegensatz herzustellen, der so in der Wirklichkeit kaum nachweisbar sein dürfte (vgl. H. Glück 1987: 6f.). Auf der anderen Seite die Tendenz, die verschiedenen Funktionen so zusammenzufügen, daß die Vorstellung einer Totalität von Sprachfunktionen entsteht. Den beiden Tendenzen liegt eine Prämisse zugrunde, von der im folgenden die Rede sein soll.

\subsection{Die Grundlage der Unterscheidung}

Die Prämisse läßt sich etwa so in Worte fassen: die Unterscheidung von schriftlicher und mündlicher Sprache ist eine Differenzierung innerhalb ein und derselben Sprache. Sie begründet nicht zwei unterschiedliche Sprachen, sondern zwei Varietäten ein und derselben.

Wenn Josef Vachek zwischen schriftlicher und mündlicher Sprache unterscheidet, dann berücksichtigt er, ohne daß dies explizit gesagt würde, nur die Verhältnisse in solchen Sprachgemeinschaften, in denen in ein und derselben Sprache geschrieben und gesprochen wird. Die verschiedenen Arten diglottischer Situationen, von denen oben die Rede war, werden nicht berücksichtigt. So spricht Vachek nur gelegentlich von ,geschriebener und gesprochener Sprache". Stattdessen zieht er es vor, die in der Tat treffenderen Bezeichnungen „geschriebene und gesprochene Norm“ zu gebrauchen. Ein solcher Sprachgebrauch setzt voraus, daß es sich um zwei Normen ein und derselben Sprache 
handelt. So wird von einem kompetenten Sprachbenutzer, „der zu einer kultivierten Sprachgemeinschaft gehört“, erwartet, daß er in der Lage ist, „,beide Normen der betreffenden Sprache gleich gut (zu) beherrschen“ (1973/1976: 246). Daß ein Sprachbenutzer auch dann seine Kompetenz unter Beweis stellen kann, wenn er gleich gut in einer Sprache spricht und in einer anderen schreibt, ist nicht bedacht worden. So werden die Sprachen der Welt, die zur gesprochenen Norm noch eine weitere schriftliche ausgebildet haben, als Sprachen qualifiziert, die die ihnen ,inhärente funktionale Kapazität“ (1973: 49) voll ausgeschöpft haben. Denn

ohne Frage ist das Ziel, auf das die Sprachentwicklung jeder Sprachgemeinschaft gerichtet ist, die höchstmögliche Effektivität sprachlicher Kommunikation und die maximale Entwicklung ihrer funktionalen Reichweite (1973/1976: 247).

Die Grundlage der Differenzierung ist also in der funktionalistischen Konzeption die gleiche wie in der glossematischen Konzeption.

Die Beschränkung auf die Verhältnisse innerhalb ein und derselben Sprache könnte der Schlüssel zur Erklärung der verschiedenen Zwänge sein, in die die funktionalistische Konzeption geraten ist. Sie erklärt die Notwendigkeit, die unterschiedlichsten Funktionen schriftlicher Äußerungen über einen Leisten zu schlagen (vgl. 2.1.), weil nur dann ein Kontrast zur mündlichen Sprache herzustellen war, wenn sich diese Funktionen auf einen gemeinsamen Nenner bringen ließen. Sie erklärt die Notwendigkeit, die Differenzen zwischen schriftlicher und mündlicher Sprache, die kaum in Abrede zu stellen sind, so zu fassen, daß aus ihnen konträre Gegensätze werden (vgl. 2.2.); denn nur eine solche Polarisierung der Begriffe war in der Lage, die ganze Last einer rein funktionalen Unterscheidung von schriftlicher und mündlicher Sprache zu tragen. Und schließlich erklärt sie die Notwendigkeit, den komplementären oder gar oppositiven Charakter des Verhältnisses beider Sprachnormen über die Maßen zu betonen. Denn nur dann, wenn die zuvor getrennten und einander entgegengesetzten Funktionen sich zu einem Ganzen zusammenfügen ließen, war die Einheit der Sprache gewährleistet (vgl. 2.3.).

Die Beschränkung auf die Verhältnisse in ein und derselben Sprache ist umso auffälliger, als es nicht den geringsten Anlaß gibt, im Rahmen einer funktionalistischen Theorie nicht auch den Fall vorzusehen, daß Menschen in einer Sprache sprechen und in einer ganz anderen Sprache schreiben, und sich damit die Funktionen, die dem Sprechen und dem Schreiben innerhalb einer Sprachgemeinschaft zukommen, auf verschiedene Sprachen verteilen. Hätte man diesen Fall bedacht, dann wären die Schwierigkeiten zu vermeiden gewesen und die Theorie insgesamt wohl auch anders angelegt worden.

Vermag man die Beschränkung der funktionalistischen Konzeption auf die Verhältnisse in ein und derselben Sprache noch aus einer verständlichen, darum aber nicht zu rechtfertigenden eurozentristischen Perspektive ableiten, der fast alle Sprachwissenschaftler gehuldigt haben (vergleichbar der „Tyrannei des Alphabets“, von der Roy Harris 1986 spricht), so ist eine weitere Beschrän- 
kung der Untersuchungen von Josef Vachek nicht mehr erklärlich - eine Art von freiwilliger Selbstbeschränkung. Von einer Sprachtheorie, in der eine schriftliche von einer mündlichen Sprachnorm unterschieden wird, wäre zu erwarten gewesen, daß sie anders als die glossematische Theorie, die dazu grundsätzlich nicht in der Lage war, nach strukturellen Unterschieden auf allen Ebenen der Sprachbeschreibung gesucht hätte, nicht nur auf der phonographischen Ebene, wie es Tradition ist. Diese Theorie hätte es nicht nur erlaubt, sondern auch nahegelegt. Tatsächlich aber hat Josef Vachek in den verschiedenen Schriften, die er zur geschriebenen Sprache, vor allem des Englischen, vorgelegt hat, nur die strukturellen Abweichungen auf der phonographischen Ebene behandelt, also Fragen der Orthographie. Auch das hat dann also die funktionalistische Konzeption mit der glossematischen gemeinsam, mit dem Unterschied allerdings, daß eine solche Selbstbeschränkung in der funktionalistischen Konzeption nicht notwendig gewesen wäre.

\section{Eine pragmatische Konzeption}

Die pragmatische Konzeption verdankt wesentliche Einsichten der funktionalistischen Konzeption und kann in gewisser Weise als deren Fortführung oder - besser - als deren Richtigstellung betrachtet werden.

\subsection{Grundlage}

Grundlage der Unterscheidung von schriftlicher und mündlicher Sprache ist in dieser Konzeption die Zusammengehörigkeit von beiden, die nicht erst durch sprachliche Korrespondenzen, sondern immer schon durch die Personen hergestellt wird, die lesen und schreiben, seien es Individuen, Gruppen von Menschen oder ganze Völker und Nationen. Eben dieser Bezug auf Personen rechtfertigt es, von einer pragmatischen Konzeption zu sprechen.

Sowohl die glossematische als auch die funktionalistische Konzeption lassen den Bezug auf Personen vermissen. Sie beziehen sich ausschließlich auf die Sprache, in der gesprochen und geschrieben wird, und erweisen sich damit als zu eng, um alle Verhältnisse erfassen zu können. Dieser Beschränkung unterliegt die pragmatische Konzeption nicht, und damit kann sie der Tatsache Rechnung tragen, daß auch die Menschen, die in einer Sprache sprechen und in einer anderen schreiben, sowohl über eine mündliche als auch über eine schriftliche Sprache verfügen, auch wenn in einem solchen Falle diese nicht als „schriftliche Form“ oder ,schriftliche Norm“ zu der von ihnen gesprochenen Sprache gelten kann.

Der Bezug auf die Personen, die sprechen und schreiben, ist nicht neu. Man findet in den Ausführungen zur funktionalistischen Konzeption mehrfach Hinweise auf eine „Sprachgemeinschaft" oder auf die „Glieder einer Sprachgemeinschaft“. Etwa, wenn man liest:

Man muß also die Schrift- und Sprechnorm für koordinierte Größen halten, (...) deren Zusammengehörigkeit nur der Tatsache zuzuschreiben ist, daß sie von einer 
und derselben Sprachgemeinschaft in komplementären Funktionen benutzt werden (J. Vachek 1939/1976: 235).

Nina Catach glaubte deshalb feststellen zu können, daß die Frage nach der schriftlichen Norm von Josef Vachek ,von einem soziolinguistischen Standpunkt aus" erörtert worden sei (N. Catach 1987: 164). Sie übersieht dabei aber, daß der Bezug auf Personen in der funktionalistischen Konzeption nicht systematisch ausgewertet worden ist. In jeder funktionalistischen Theorie wird - mehr oder weniger explizit - auf die Größen Bezug genommen, die Träger der Funktionen sind, und so nimmt es nicht wunder, wenn auch in einer funktionalistischen Sprachtheorie auf die Bedürfnisse von Menschen Bezug genommen wird, die mithilfe schriftlicher oder mündlicher Äußerungen auf solche Bedürfnisse reagieren. Ein solcher Bezug wird aber in der Konzeption von Josef Vachek nicht expliziert. Er bleibt folgenlos und erweist sich somit für diese Theorie als nicht konstitutiv.

In vielen Sprachgemeinschaften der Welt ist die Zusammengehörigkeit von schriftlicher und mündlicher Sprache nicht auf den sozialen Bezug beschränkt. Zu diesem kann, wie jedermann weiß, ein sprachlicher hinzukommen, und zwar immer dann, wenn die Menschen, die sprechen und schreiben, dies in ein und derselben Sprache tun. Schriftliche und mündliche Sprache sind unter solchen Umständen also zweifach aufeinander bezogen: einmal durch die Personen und dann auch noch durch die Korrespondenzen, die sich zwischen schriftlicher und mündlicher Sprache ergeben. Anzunehmen, daß beide dann auch den gleichen Rang beanspruchen könnten, wäre unberechtigt. Der Fall, daß schriftliche und mündliche Sprache durch die Menschen, die sich ihrer bedienen, aufeinander bezogen sind, gilt überall auf der Welt. Es ist grundlegend und unabdingbar für die Bestimmung von schriftlicher und mündlicher Sprache. Der andere Fall, daß dazu auch noch in ein und derselben Sprache gesprochen und geschrieben wird, ist zwar recht häufig, zur Bestimmung von schriftlicher und mündlicher Sprache aber nicht unbedingt erforderlich.

Wenn schriftliche und mündliche Sprache durch die Menschen, die von ihnen Gebrauch machen, aufeinander bezogen sind und in vielen Fällen darüber hinaus auch noch über ihre Form, dann stellt sich die Frage, auf welcher Dimension sie dann voneinander unterschieden werden können.

\subsection{Die Unterscheidung}

Der Grundgedanke der Funktionalisten läuft darauf hinaus, schriftliche und mündliche Sprache nach ihren Funktionen zu unterscheiden. Ich denke, daß dieser Gedanke richtig ist, nur muß er anders gefaßt werden.

Zunächst einmal ist klarzustellen: es geht hier nicht um Funktionen einzelner, konkreter Schriftäußerungen (Texte), auch nicht um Funktionen ganzer Textsorten. Und ob es Sinn macht, nach der einen Funktion aller Schriftäußerungen zu suchen, erscheint mir höchst fragwürdig zu sein. Wenn 
schriftliche und mündliche Sprache nach ihren Funktionen unterschieden werden sollen, dann geht es einzig und allein um die Funktion, die der schriftlichen Sprache zukommt.

Wenn man sich dies klargemacht hat, dann dürfte es auch nicht schwerfallen, sich mit einem im Grunde recht banalen Gedanken vertraut zu machen. Schriftliche Sprache ist die Sprache, deren Beherrschung beim Lesen und vor allem beim Schreiben vorausgesetzt wird, und zwar unabhängig davon, ob in dieser Sprache auch gesprochen wird. Die schriftliche Sprache spielt also im Prozeß der Produktion und Rezeption schriftlicher Texte eine Rolle. Dieser Tatsache ist bei der Bestimmung der Funktion schriftlicher Sprache Rechnung zu tragen. Sie bildet ihr Fundament. Wir können dann definieren: Die Aufgabe oder die Funktion schriftlicher Sprache besteht darin, die sprachlichen Mittel zur Verfügung zu stellen, die bei der Produktion und Rezeption schriftlicher Äußerungen (Texte) benötigt werden.

Eine solche Definition berücksichtigt, daß in ihr alle notwendigen Elemente einer Funktionsbestimmung enthalten sind:

- der Träger der Funktion - hier: die schriftliche Sprache

- das übergeordnete System, im Hinblick auf welches der Träger eine Funktion ausübt - hier: der Prozeß der Produktion und Rezeption schriftlicher Äußerungen (Texte)

- die Art und Weise, in der die Funktion wirksam wird - hier: die Bereitstellung der jeweils notwendigen sprachlichen Mittel.

Eine Bestimmung der Funktion schriftlicher Sprache, die auf Lesen und Schreiben und also auf Aktivitäten von Menschen Bezug nimmt, hat zudem den Vorteil, daß sie ohne weiteres auf die Grundlage angewendet werden kann, durch die die Zusammengehörigkeit von schriftlicher und mündlicher Sprache gewährleistet ist (vgl. 3.1.). Das eine ergibt sich aus dem anderen.

\subsection{Der Geltungsbereich der Unterscheidung}

Die Frage, wie weit der Bereich zu fassen ist, in dem die Differenzierung Gültigkeit hat: ob er sich auf den phonographischen Bereich beschränkt, wie die Glossematiker und auch Josef Vachek voraussetzen, oder nicht doch auf weitere Ebenen der Sprachbeschreibung auszudehnen ist (zumindest auf die morphologische, syntaktische oder lexikalische Ebene), diese Frage läßt sich nicht mit einem Wort beantworten.

Sie ist klar und eindeutig zu beantworten für alle Gemeinschaften, die in einer Sprache schreiben und in einer anderen reden, also für alle Fälle von Diglossie mit Bilingualismus. Für sie ist die Unterscheidung auf allen Ebenen und in allen Bereichen der Sprache selbstverständlich. Schriftliche und mündliche Sprache sind dann in der Tat zwei verschiedene Sprachen. Zu einem Problem wird die Unterscheidung aber immer dann, wenn nicht in zwei grundsätzlich verschiedenen, sondern in ein und derselben Sprache geschrieben und gesprochen wird, eine Reihe von diglottischen Situationen eingeschlossen. 
Man wird gut daran tun, in diesen Fällen von der Vorstellung eines Kontinuums auszugehen, dessen einer Pol durch Fälle von Diglossie mit Bilingualismus, wie sie oben angesprochen worden sind, und dessen anderer Pol etwa durch eine gelungene phonetische Transkription besetzt werden (zur phonetischen Transkription vgl. J. Vachek 1949/1967). Auf einem solchen Kontinuum hätten wir dann mit allen Möglichkeiten von Übergängen zu rechnen:

- mit minimalen Abweichungen, den sog. „Akzidentien“,

- mit Divergenzen, die auf die eine oder andere Ebene beschränkt bleiben,

- mit größeren Abweichungen im Bereich der Zahlen etwa bis hin zu diastratisch oder diachronisch bedingten Disproportionen, wie sie für die deutschsprechende Schweiz einerseits und die arabischen Länder und China andererseits nachweisbar sind (vgl. 1.2.).

Für jede einzelne Sprache wäre dann zu prüfen, auf welchem Punkt der Skala sie zu lokalisieren ist.

Die Ausführungen zur schriftlichen Sprache haben einige systematische Konsequenzen, auf die ich abschließend eingehen möchte.

\subsection{Einige Konsequenzen}

1. Form und Funktion sind, wie auch sonst in der Sprachbeschreibung, strikt auseinanderzuhalten. Wenn gesagt wurde, daß der Begriff der schriftlichen Sprache ein pragmatischer Begriff sei, dann heißt das, daß er primär funktional zu fassen ist. Eine Person, eine Gruppe von Personen oder eine ganze Sprachgemeinschaft verwenden eine bestimmte Sprache bei der Produktion und Rezeption schriftlicher Äußerungen, sei es die Sprache, die sie auch sonst verwenden, sei es eine andere Sprache. Der Begriff der schriftlichen Sprachform dagegen ist kein funktionaler, sondern ein strukturlinguistischer Begriff. Er referiert auf die sprachliche Form, unbeschadet der Tatsache, wer sich dieser Sprache bedient und zu welchen Zwecken. Die schriftliche Form des Chinesischen bleibt beispielsweise die schriftliche Form des Chinesischen und wird nicht etwa zur schriftlichen Form des Japanischen, wenn es Japaner gegeben hat, die sich ihrer beim Schreiben bedienten. Der Begriff der schriftlichen Sprache läßt den Bezug auf die sprachliche Form, der Begriff der schriftlichen Sprachform den auf die Personen und Zwecke ausgespart.

2. Der einfachste und klarste Fall schriftlicher Sprache liegt vor, wenn Menschen in einer Sprache sprechen und in einer anderen schreiben: ein Fall von Diglossie mit Bilingualismus, von dem schon mehrfach die Rede war. In einem solchen Fall handelt es sich um eine Sprache, die nicht nur ihrer Funktion, sondern auch ihrer Form nach grundsätzlich von der mündlichen Sprache unterschieden ist. Die Frage stellt sich, welche systematische Bedeutung diesen Fällen von Diglossie mit Bilingualismus zukommt. Die Frage stellt sich umso mehr, als unter den Sprachgemeinschaften, die die Aufmerksamkeit der Sprachwissenschaftler bisher in besonderer Weise auf sich gezogen haben, 
solche Fälle eher selten sind. Ich denke, daß ihre Bedeutung nicht hoch genug veranschlagt werden kann. Sie zeigen, unter welchen Bedingungen generell eine Bestimmung der schriftlichen Sprache möglich ist und unter welchen besonderen Bedingungen eine solche Bestimmung sowohl die Funktion als auch die Form dieser Sprache berücksichtigen kann. Der Begriff der schriftlichen Sprache ist also seiner Extension nach weiter als der der schriftlichen Sprachform. Er enthält diesen als einen besonderen Fall.

3. Damit wird der Begriff der schriftlichen Sprachform aber nicht überflüssig. Im Gegenteil! Er ist zwar in seinen Anwendungsmöglichkeiten eingeschränkt, da er nur für Sprachgemeinschaften Verwendung finden kann, in denen in ein und derselben Sprache gesprochen und geschrieben wird. Doch wird mit ihm ein Sachverhalt erfaßt, der im Begriff der schriftlichen Sprache nicht in jedem Fall enthalten ist - die Tatsache nämlich, daß die Sprache, die beim Schreiben verwendet wird, ihrer Form nach Entsprechungen zu der Sprache aufweist, in der sonst auch gesprochen wird. Diese Eigenschaft läßt ihn nützlich werden bei der Beschreibung aller Sprachen ohne Diglossie, also etwa bei der Beschreibung fast aller europäischer Sprachen. Eine solche Fassung des Begriffs setzt aber voraus, daß die glossematische Vorstellung, daß allein die Form der Ausdrucksebene und nicht auch die Form der Inhaltsebene betroffen sei, aufgegeben wird. Wenn man nicht von vornherein und nicht ohne Not den Begriff der schriftlichen Sprachform auf sprachliche Entsprechungen der phonographischen Ebene beschränken will, wird man ihn zumindest bis zum Nachweis des Gegenteils - auf alle Ebenen der Sprachbeschreibung beziehen müssen.

4. Die Frage, ob dann, wenn eine Sprache in zwei Sprachformen vorliegt, geschlossen werden kann, daß es sich um zwei Varianten handele, denen ein und dieselbe Sprache oder ein und dasselbe Sprachsystem zugrundeliegt, scheint mir problematisch zu sein. Unproblematisch ist es, wenn man Objektund Modellbereich unterscheidet und für den Modellbereich eine einheitliche Beschreibung vorsieht (vgl. H. Glück 1987: 37). Abweichungen ließen sich ohne weiteres als Ausnahmen, Besonderheiten oder medial bedingte Varianten kennzeichnen. Unproblematisch wäre eine solche Annahme in jedem Falle, wenn sich nachweisen ließe, daß, womit die glossematische Sprachtheorie allein rechnet, die Abweichungen sich auf die Form der Ausdrucksebene beschränken und nicht auf die Form der Inhaltsebene durchschlagen. Ein solcher Nachweis steht aber noch aus, und es ist recht unwahrscheinlich, daß er überhaupt geführt werden kann. So scheinen mir alle Aussagen über den Objektbereich, die von der Annahme eines zugrundeliegenden und sowohl für die schriftliche als auch für die mündliche Sprache einheitlichen Sprachsystems ausgehen, kaum zu rechtfertigen zu sein. Das hat auch Josef Vachek so gesehen, wenn auch seine Begründung an einem anderen Punkt ansetzt:

Wir sehen also, daß eine vollkommen analoge Struktur von Schrift- und Sprechnorm in keiner Sprachgemeinschaft vorhanden ist. Daraus folgt natürlich, daß man das Vorhandensein einer universalen abstrakten Norm, die der Schrift- und 
Sprechnorm übergeordnet wäre, ausschließen muß, und zwar für alle existierenden Sprachen. Wenn man nämlich ihre Existenz selbst in solchen Sprachen nicht anerkennen kann, wo das phonologische Prinzip des Alphabets so günstige Voraussetzungen bietet, so kann man ihr Vorhandensein noch weniger in solchen Sprachen ansetzen, welche bei dem Aufbau ihrer Schriftnormen vom phonologischen Prinzip beträchtlich abgewichen sind oder sich ganz anderer Prinzipien bedienen (...). Man muß also die Schrift- und Sprechnorm für koordinierte Größen halten, denen keine höhere Norm übergeordnet ist und deren Zusammengehörigkeit nur der Tatsache zuzuschreiben ist, daß sie von einer und derselben Sprachgemeinschaft in komplementären Funktionen benutzt werden (ebd.: S. 235).

5. Man kann die vorliegenden Ausführungen als einen Beitrag zur Klärung linguistischer Begriffe nehmen. Das wäre zwar nicht ganz falsch, würde aber genau das außer Acht lassen, worauf es mir angekommen ist. Zweck meiner Ausführungen ist es, die theoretischen Voraussetzungen dafür zu schaffen, daß das selbstverordnete Verbot, oberhalb der phonographischen Ebene nach Formen schriftlicher Sprache zu suchen, aufgehoben wird und damit die Untersuchung schriftlicher Sprache allererst ihren Anfang nehmen kann.

\section{Literatur}

Burke, Peter: Küchenlatein, Sprache und Übergangssprache in der frühen Neuzeit, Berlin: Wagenbach (1. Aufl. 1987), 2. Aufl. 1989.

Catach, Nina: New Linguistic Approaches to a Theory of Writing. In: Simon, P. und X. Battestini (Hgg.): Developments in Linguistics and Semiotics. Washington 1987, S. $161-174$.

Clanchy, M. T.: From Memory to Written Record, England 1066-1307, London: Arnold 1979.

Coulmas, Florian: Über Schrift (stw 378), Frankfurt a. M. 1981.

Ders.: Writing Systems of the World, Oxford: Blackwell 1989.

Engel, Ulrich: Deutsche Grammatik, Heidelberg: Julius Groos 1988.

Glück, Helmut: Schrift und Schriftlichkeit, Stuttgart: Metzler 1987.

Goody, Jack: The interface between the written and the oral, Cambridge: Univ. Press 1987.

Günther, Hartmut: Schriftliche Sprache (Konzepte der Sprach- und Literaturwissenschaft 40), Tübingen: Niemeyer 1988.

Haas, William: On the Writing of Numbers. In: Catach, Nina (Hg.): Pour une Theorie de la Langue Ecrite, Paris 1988, S. 203-212.

Hjelmslev, Louis: Prolegomena to a Theory of Language (1. Aufl. 1943), Madison: The Univ. of Wisconsin Press 1961.

Ifrah, Georges: Universalgeschichte der Zahlen, Frankfurt/New York: Campus 1989.

Keller, R.E.: Diglossia in German-Speaking Switzerland. In: Haas, William (Hg.): Standard Languages, Spoken and Written, Cambridge: Univ. Press 1982, S. 70-93.

Klein, Wolfgang: Gesprochene Sprache - geschriebene Sprache, Lili 59 (1985), 9-35.

Klopsch, Paul: Latein als Literatursprache. In: Propyläen Geschichte der Literatur, Bd. 2: Die mittelalterliche Welt, 600-1400, Berlin 1982, S. 310-334.

Kohrt, Manfred: Theoretische Aspekte der deutschen Orthographie. Tübingen: Niemeyer 1987.

Kühn, Peter: Deutsche Sprache in der Schweiz. In: Lexikon der Germanistischen Linguistik, 2. Aufl. Tübingen: Niemeyer 1980, S. 531-536.

Li, Charles N. und Sandra A. Thompson: The Gulf Between Spoken and Written Language: A Case Study in Chinese. In: Tannen, Deborah (Hg.): Spoken and Written Language, Norwood: Ablex 1982, S. 77-88. 
Ludwig, Otto: Einige Vorschläge zur Begrifflichkeit und Terminologie in Untersuchungen im Bereich der Schriftlichkeit. In: Günther, Klaus B. und Hartmut Günther (Hgg.): Schrift, Schreiben, Schriftlichkeit (Germanistische Linguistik 49), Tübingen: Niemeyer 1983, S. $1-15$.

Ders.: Zur Frage der Autonomie und Abhängigkeit geschriebener Sprache, erörtert am Beispiel schriftlicher Textformen. In: Linguistische Studien, Reihe A, Arbeitsberichte 173, Berlin 1988, S. 36-51.

Lüdi, Georges: Französisch: Diglossie und Polyglossie (im Druck).

Mitchell, T.F.: More than a matter of "writing with the learned, pronouncing with the vulgar". Some preliminary observations on the Arabic koine. In: Haas, William (Hg.): Standard Languages, Written and Spoken, Cambridge: Univ. Press 1982, S. $123-155$.

Sieber, Peter und Horst Sitta: Mundart und Standardsprache als Problem der Schule, Aarau, Frankfurt, Salzburg: Sauerländer 1986.

Uldall, H. J.: Speech and Writing, Acta Linguistica 4 (1949), S. 11-16.

Vachek, Josef: Zum Problem der geschriebenen Sprache (zuerst 1939). In: Scharnhorst u. a. (Hgg.): Grundlagen der Sprachkultur und Sprachpflege, Teil 1, Berlin 1973, S. $229-239$.

Ders.: Written Language and Printed Language (zuerst 1948). In: Vachek, Josef (Hg.): A Prague School Reader in Linguistics, Bloomington 1964, S. 453-460.

Ders.: Some Remarks on Writing and Phonetic Transcription (zuerst 1949). In: Hamp, Eric u. a. (Hgg.): Reading in Linguistics II, Chicago und London, 2. Aufl. 1967, S. $152-157$.

Ders.: On the linguistic Status of Written Utterances. In: Omagiu Lui Alexandru Rosetti La 70 De Ani, Bucaresti 1965, 959-963.

Ders.: The present State of Research in Written Language, Folia Linguistica 6 (1973), S. $47-60$.

Ders.: Geschriebene Sprache. Allgemeine Probleme und Probleme des Englischen. In: Scharnhorst, J. und E. Ising (Hgg.): Grundlagen der Sprachkultur, Teil 1, Berlin 1976, S. $240-295$.

Ders.: Paralinguistic Sounds, Written Language and Language Development, Folia Historica 2 (1981), S. 179-190.

Ders.: English Orthography. In: Haas, William (Hg.): Standard Languages, Spoken and Written, Manchester: Univ. Press 1982, S. 37-56.

Adresse des Verfassers: Prof. Dr. Otto Ludwig, Universität Hannover, Seminar für Deutsche Literatur und Sprache, Welfengarten 1, D-W-3000 Hannover 1. 